\title{
CLASSIFICATION OF LIMITS OF TRIANGULAR MATRIX ALGEBRAS
}

\author{
by A. HOPENWASSER and S. C. POWER
}

(Received 21st February 1991)

\begin{abstract}
Let $T_{n}$ be the operator algebra of upper triangular $n \times n$ complex matrices. Three families of limit algebras of the form $\lim \left(T_{n_{k}}\right)$ are classified up to isometric algebra isomorphism: (i) the limit algebras arising when the embeddings $T_{n_{k}} \rightarrow T_{n_{k}+1}$ are alternately of standard and refinement type; (ii) limit algebras associated with refinement embeddings with a single column twist; (iii) limit algebras determined by certain homogeneous embeddings. The last family is related to certain fractal like subsets of the unit square.
\end{abstract}

1991 Mathematics subject classification: 47 D25.

\section{Introduction}

The isomorphism class of a direct limit of full matrix algebras (a UHF $\mathrm{C}^{*}$-algebra) is completely determined by the supernatural number associated with the sequence of ranks of the matrix algebras ([3]). A number of recent papers $[1,4,5,6,8,9,10,12,13$, 14] have been devoted to non-self-adjoint subalgebras of UHF algebras (and, more generally, of $\mathrm{AF} \mathrm{C}^{*}$-algebras). Usually, the algebras in question contain a canonical masa in the sense of Stratila and Voiculescu [11]; often they are triangular: the intersection with the adjoint algebra is the canonical masa. The purpose of this note is to give a complete classification of three particular families of triangular subalgebras of UHF algebras: The first family consists of those algebras which arise as the direct limit of a system of upper triangular matrix algebras, where the embeddings are alternately the refinement and the standard embeddings. The second family arises from refinement embeddings with a twist (cf. [5]), and the third family is a class of homogeneous nest subalgebras related to fractal subsets of the unit square.

For all classifications we use the topologized fundamental relation, which we believe to be an effective and intuitive discriminant.

For the first family it will be convenient to write the full matrix algebras in the direct systems which arise as tensor products; so our systems will have the form

$$
M_{k_{1}} \rightarrow M_{k_{1}} \otimes M_{k_{2}} \rightarrow M_{k_{1}} \otimes M_{k_{2}} \otimes M_{k_{3}} \rightarrow \cdots
$$

Upper triangular subalgebras, $T_{k_{1} k_{2} \ldots k_{n}}$, in $M_{k_{1}} \otimes \cdots \otimes M_{k_{n}}$ are specified by giving a total order to the minimal projections in the natural diagonal algebra $D_{k_{1}} \otimes \cdots \otimes D_{k_{n}}$, 
where for each $k, D_{k}$ is the algebra of diagonal matrices in $M_{k}$. Let $[k]$ denote the set $\{1,2, \ldots, k\}$ and suppose that $\left\{e_{i} \mid i \in\left[k_{m}\right]\right\}$ are the minimal projections of $D_{k_{m}}$, totally ordered according to the order on the index set. Then the set of minimal projections of $D_{k_{1}} \otimes \cdots \otimes D_{k_{n}}$ is $\left\{e_{i_{1}} \otimes \cdots \otimes e_{i_{n}} \mid i_{m} \in\left[k_{m}\right], m=1, \ldots, n\right\}$. Totally order this set by the lexicographic order on the natural index set $\pi_{m=1}\left[k_{m}\right]$; this yields the order with respect to which $T_{k_{1} k_{2} \ldots k_{m}}$ is the algebra of upper triangular matrices.

Throughout this paper we fix one set of canonical matrix units for each algebra $M_{k_{1}} \otimes \cdots \otimes M_{k_{n}}$. These matrix units will be indexed by pairs of multi-indices $i, j$ in $\pi_{m=1}^{n}\left[k_{m}\right]:$ if $i=\left(i_{1}, \ldots, i_{n}\right)$ and $j=\left(j_{1}, \ldots, j_{n}\right)$ then $e_{i j}=e_{i_{1} j_{1}} \otimes \cdots \otimes e_{i_{n} j_{n}}$. Note that $e_{i j} \in T_{k_{1} k_{2} \ldots k_{n}}$ if, and only if, $i \leqq j$ (lexicographic order).

Now suppose that the (UHF) $C^{*}$-algebra $B$ is given as the direct limit of the system

$$
M_{k_{1}} \rightarrow M_{k_{1}} \otimes M_{k_{2}} \rightarrow M_{k_{1}} \otimes M_{k_{2}} \otimes M_{k_{3}} \rightarrow \cdots
$$

with unital embeddings which carry the diagonal algebras into diagonal algebras and the upper triangular algebras into upper triangular algebras. Then this yields subsystems

$$
D_{k_{1}} \rightarrow D_{k_{1}} \otimes D_{k_{2}} \rightarrow D_{k_{1}} \otimes D_{k_{2}} \otimes D_{k_{3}} \rightarrow \cdots
$$

and

$$
T_{k_{1}} \rightarrow T_{k_{1} k_{2}} \rightarrow T_{k_{1} k_{2} k_{3}} \rightarrow \cdots
$$

with limits $D$ and $T$ respectively. We have $D \subseteq T \subseteq B, D$ is a (regular) canonical masa in $B$, and $T \cap T^{*}=D$.

There are many isomorphism types of such canonical triangular algebras. Indeed, certain UHF algebras contain uncountably many distinct types in each of the three families under consideration.

The two embeddings used in the first family are the refinement embedding, $\rho$, and the standard embedding, $\sigma$, given by

$$
\rho_{t}: M_{k} \rightarrow M_{k} \otimes M_{t}:\left[a_{i j}\right] \rightarrow\left[a_{i j} I_{t}\right]
$$

and

$$
\sigma_{t}: M_{k} \rightarrow M_{k} \otimes M_{t}: a \rightarrow a \oplus \cdots \oplus a \quad \text { ( } t \text { factors) }
$$

The positive integer $t$ is the multiplicity of the embedding. Note that $\sigma_{t}=A d U_{\pi} \circ \rho_{t}$ for a suitable unitary operator $U_{\pi}$ induced by a permutation $\pi$ of the minimal projections in $D_{k} \otimes D_{t}$

The action of $\rho_{k_{n+1}}$ on the matrix units of $M_{k_{1}} \otimes \cdots \otimes M_{k_{n}}$ may be described in terms of indices as follows: the matrix unit bearing the indices $\left(i_{1}, \ldots, i_{n}\right)$ and $\left(j_{1}, \ldots, j_{n}\right)$ is carried to the sum of all those matrix units in $M_{k_{1}} \otimes \cdots \otimes M_{k_{n+1}}$ with indices $\left(i_{1}, \ldots, i_{n}, h\right)$ and $\left(j_{1}, \ldots, j_{n}, h\right)$, where $h \in\left[k_{n+1}\right]$.

The permutation $\pi$ for which $\sigma_{k_{n+1}}$ can be expressed as $A d U_{\pi} \circ \rho_{k_{n+1}}$ is obtained via a 
process of "reverse lexicography": the set $\left[k_{n+1}\right] \times\left[k_{1}\right] \times \cdots \times\left[k_{n}\right]$ with the lexicographic order is totally ordered and has the same cardinality as $\left[k_{1}\right] \times \cdots \times\left[k_{n}\right] \times$ $\left[k_{n+1}\right]$. Let $\phi:\left[k_{n+1}\right] \times\left[k_{1}\right] \times \cdots \times\left[k_{n}\right] \rightarrow\left[k_{1}\right] \times \cdots \times\left[k_{n}\right] \times\left[k_{n+1}\right]$ be the unique order isomorphism between these two sets. Define $\pi$ of the minimal projection with index $\left(i_{1}, \ldots, i_{n}, i_{n+1}\right)$ in $D_{k_{1}} \otimes \cdots \otimes D_{k_{n+1}}$ to be the minimal projection with index $\phi\left(i_{n+1}, i_{1}, \ldots, i_{n}\right)$.

The classification of the family of direct limits of type

$$
T_{k_{1}} \stackrel{\sigma}{\rightarrow} T_{k_{1} k_{2}} \stackrel{\sigma}{\rightarrow} T_{k_{1} k_{2} k_{3}} \stackrel{\sigma}{\rightarrow}
$$

appeared in $[1,5,8]$, while classification of the corresponding family with $\rho$ in place of $\sigma$ appeared in $[5,8]$. For each family, the supernatural number associated with the sequence $k_{1}, k_{2}, k_{3}, \ldots$ is a complete invariant, i.e. two algebras from the same family are isometrically isomorphic if, and only if, their associated supernatural numbers are equal. By the way it is evident that no refinement limit can be isomorphic to a standard limit: the refinement limits are all nest subalgebras of the ambient UHF algebra while the standard limits possess no invariant projections.

One direction of the classification result cited above follows from [8, Theorem 6.14]:

Theorem 1. Let $D_{i} \subseteq T_{i} \subseteq B_{i}, i=1,2$, where $B_{i}$ is a $U H F$ algebra, $D_{i}$ is a (regular) canonical masa, and $T_{i}$ is a closed subalgebra of $B_{i}$ (or even a closed $D_{i}$-bimodule) which generates $B_{i}$ as a $C^{*}$-algebra. If $T_{1}$ and $T_{2}$ are isometrically isomorphic than $B_{1}$ and $B_{2}$ are isomorphic $C^{*}$-algebras.

This theorem will be useful in the classification of the $\rho \sigma$-alternation algebras. See also [5, Theorem 3.26 and 2, Theorem 3.3] for a similar result.

Another major tool in this classification will be the fundamental relation introduced in [8]. Its value lies in the fact that it acts as a complete invariant for triangular subalgebras of $\mathrm{AF} \mathrm{C}^{*}$-algebras [9]. Given a triple $D \subseteq T \subseteq B$ as above, the fundamental relation will be a topologized relation defined on the maximal ideal space $M(D)$ of $D$ in terms of normalizing partial isometries in $T$. A partial isometry, $v$, in $T$ is normalizing if $v D v^{*} \subseteq D$ and $v^{*} D v \subseteq D$. For elements $x$ and $y$ in $M(D)$, viewed as states on $D$, define $y R(T) x$ if, and only if, there is a normalizing partial isometry $v \in T$ such that $x(d)=y\left(v d v^{*}\right)$, for all $d \in D$. [8, Lemma 6.3].

We shall need to specify the fundamental relation for the $\rho \sigma$-algebras; for completeness, we will do the same for the pure refinement and pure standard algebras, although these are in the literature [8]. The task is made simpler by the fact that it is sufficient to use the canonical set of matrix units for $T$ in place of all normalizing partial isometries, see [9].

If $e$ is a matrix unit, let $E=\{(y, x) \mid y R(T) x$ via the matrix unit $e\}$. The fundamental relation is topologized by taking all such sets $E$ as a base for a topology on $R(T)$. It turns out that the basic sets, $E$, are compact in this topology, and this will be useful 
later in classifying the twisted refinement embeddings. This topology is not the same as the relative product topology obtained from the Gelfand topology on $M(D)$. The main theorem in [9] states, amongst other things, that two canonical triangular subalgebras of $\mathrm{AF} \mathrm{C}^{*}$-algebras are isometrically isomorphic if, and only if, their associated fundamental relations are isomorphic as topologized relations.

The first step in describing the fundamental relation is the identification of $M(D)$ with $\pi_{n=1}^{\infty}\left[k_{n}\right]$. Indeed, $x$ is completely determined by its values on the projections of $D$, each of which necessarily lies in one of the finite dimensional subalgebras $D_{k_{1}} \otimes \cdots \otimes D_{k_{n}}$. In each such subalgebra there is a unique minimal projection, say $e_{\left(x_{1}, \ldots, x_{n}\right)}=e_{x_{1}} \otimes \cdots \otimes$ $e_{x_{n}}$, at which $x$ takes the value 1 and the index coordinates are coherent from level to level; thus we may identify $x$ with an element $\left(x_{1}, x_{2}, x_{3}, \ldots\right)$ in $\pi_{n=1}^{\infty}\left[k_{n}\right]$. Conversely, each point in $\pi_{n=1}^{\infty}\left[k_{n}\right]$ determines a state on $D$ and this correspondence between $M(D)$ and $\pi_{n=1}^{\infty}\left[k_{n}\right]$ is a homeomorphism with respect to the Gelfand topology on $M(D)$ and the product topology on $\pi_{n=1}^{\infty}\left[k_{n}\right]$.

A projection of the form $e=e_{\left(i_{1}, \ldots, i_{n}\right)}$ in $D$ corresponds to a subset of $M(D)$; that subset is $\left\{x \in \pi_{n=1}^{\infty}\left[k_{n}\right] \mid x_{1}=i_{1}, \ldots, x_{n}=i_{n}\right\}$. Normalizing partial isometries, $v$, act by conjugation $e \rightarrow v e v^{*}$ on projections in $D$, and so carry certain totally ordered families of projections $\left\{e_{\left(x_{1}, \ldots, x_{n}\right)}\right\}$ corresponding to points $x$ in $\pi_{n=1}^{\infty}\left[k_{n}\right]$ into other totally ordered families; in other words, each $v$ induces a partial homeomorphism on $\pi_{n=1}^{\infty}\left[k_{n}\right]$. In particular, if $i=\left(i_{1}, \ldots, i_{n}\right)$ and $j=\left(j_{1}, \ldots, j_{n}\right)$ then the matrix unit $e_{i, j}$ has domain $\left\{x \mid x_{1}=j_{1}, \ldots, x_{n}=j_{n}\right\}$, range $\left\{y \mid y_{1}=i_{1}, \ldots, y_{n}=i_{n}\right\}$, and acts by $\left(j_{1}, \ldots, j_{n}, x_{n+1}, \ldots\right) \mapsto$ $\left(i_{1}, \ldots, i_{n}, x_{n+1}, \ldots\right)$. (This follows from repeated use of the fact that the matrix unit with indices $\left(i_{1}, \ldots, i_{n}\right)$ and $\left(j_{1}, \ldots, j_{n}\right)$ in $M_{k_{1}} \otimes \cdots \otimes M_{k_{n}}$ is carried by $\rho$ into a sum of matrix units, each with indices of the form $\left(i_{1}, \ldots, i_{n}, x_{n+1}\right)$ and $\left(j_{1}, \ldots, j_{n}, x_{n+1}\right)$.) Since the canonical matrix units determine the fundamental order, we have $y R(T) x$ if, and only if $y$ and $x$ have a common tail $\left(y_{e}=x_{e}\right.$, for all $\left.s>n\right)$ and the initial segment of $y$ for this tail, $\left(y_{1}, \ldots, y_{n}\right)$, precedes $\left(x_{1}, \ldots, x_{n}\right)$ lexicographically.

To determine the fundamental relation for the standard embeddings,

$$
T_{k_{1}} \stackrel{\sigma_{k_{2}}}{\longrightarrow} T_{k_{1} k_{2}} \stackrel{\sigma_{k_{3}}}{\longrightarrow} T_{k_{1} k_{2} k_{3}} \rightarrow \cdots
$$

it is most convenient to reindex the minimal projections in each $D_{k_{1}} \otimes \cdots \otimes D_{k_{n}}$. (In other words, we use a different isomorphism between $M(D)$ and $\pi_{n=1}^{\infty}\left[k_{n}\right]$.) Recall that $\sigma_{k_{2}}: M_{k_{1}} \rightarrow M_{k_{1}} \otimes M_{k_{2}}$ has the form $A d U_{\pi} \circ \rho_{k_{2}}$ where $\pi$ is induced from the total order isomorphism $\phi:\left[k_{2}\right] \times\left[k_{1}\right] \rightarrow\left[k_{1}\right] \times\left[k_{2}\right]$ with domain and range carrying the lexicographic order. Replace the index $\phi\left(i_{2}, i_{1}\right)$ for a minimal projection in $D_{k_{1}} \otimes D_{k_{2}}$ by $\left(i_{1}, i_{2}\right)$ itself; the minimal projections of $D_{k_{1}} \otimes D_{k_{2}}$ are again indexed by $\left[k_{1}\right] \times\left[k_{2}\right]$, but now the order is reverse lexicographic. Repetition yields the reverse lexicographic order on each of the index sets $\left[k_{1}\right] \times \cdots \times\left[k_{n}\right]$ for the minimal projections of $D_{k_{1}} \otimes \cdots \otimes D_{k_{n}}$. Furthermore, with this reindexing, once again we have that a matrix unit in $M_{k_{1}} \otimes \cdots \otimes$ $M_{k_{n}}$ with indices $i=\left(i_{1}, \ldots, i_{n}\right)$ and $j=\left(j_{1}, \ldots, j_{n}\right)$ is carried to a sum of matrix units, each with indices $\left(i_{1}, \ldots, i_{n}, x_{n+1}\right)$ and $\left(j_{1}, \ldots, j_{n}, x_{n+1}\right), x_{n+1}$ taking all values in $\left[k_{n+1}\right]$. Also 
the matrix unit $e_{i j}$ lies in $T_{k_{i} \ldots k_{n}}$ if, and only if, $i<_{r} j$ (reverse lexicographic order). Thus, we obtain $y R(T) x$ if, and only if, $y$ and $x$ have the same tails and the initial segment of $y$ preceeds the initial segment of $x$ in reverse lexicographic order.

The fundamental relation for the alternating embeddings,

$$
T_{k_{0}} \stackrel{\rho_{k_{1}}}{\longrightarrow} T_{k_{0} k_{1}} \stackrel{\rho_{k_{2}}}{\longrightarrow} T_{k_{0} k_{1} k_{2}} \rightarrow \cdots
$$

can be obtained by alternating the procedures for $\rho$ and $\sigma$, or more simply from the observation that $\sigma_{k_{2}} \circ \rho_{k_{1}}: M_{k_{0}} \rightarrow M_{k_{0}} \otimes M_{k_{1}} \otimes M_{k_{2}}$ has the form $A d U_{\pi} \circ \rho_{k_{1} k_{2}}$ where $\pi$ is induced by the isomorphism

$$
\phi:\left[k_{2}\right] \times\left[k_{0}\right] \times\left[k_{1}\right] \rightarrow\left[k_{0}\right] \times\left[k_{1}\right] \times\left[k_{2}\right],
$$

domain and range ordered lexicographically. Relabelling the indices of minimal projections as above, it follows that $y R(T) x$ if, and only if, $y$ and $x$ have the same tails and the initial segment of $y$ precedes the initial segment of $x$ in the "quasi-lexicographic" order in which the highest even index of the initial segment takes precedence and we then proceed down the even indexed terms and back up the odd indexed terms.

There is a variation of this which is notationally handy for the proof of the classification of the alternating embedding: relabel the even terms so that they carry negative indices (in reverse order, of course) and the odd terms so that they are indexed by all the positive integers. If we then consider the system

$$
\mathbb{C} I \stackrel{\rho_{r_{1}}}{\longrightarrow} T_{r_{1}} \stackrel{\sigma_{s-1}}{\longrightarrow} T_{r_{1} s_{-1}} \stackrel{\rho_{r_{2}}}{\longrightarrow} T_{r_{1} s_{-1} r_{2}} \rightarrow \cdots
$$

then $M(D)$ isomorphic to $\pi_{n=-\infty}^{-1}\left[s_{n}\right] \times \pi_{n=1}^{\infty}\left[r_{n}\right]$, and $y R(T) x$ if, and only if, $y$ and $x$ have the same double infinite tail (i.e. $y_{j}=x_{j}$ for $j<-n$ and $j>n$ ) and the "middle" segments $\left(y_{-n}, \ldots, y_{n}\right)$ and $\left(x_{-n}, \ldots, x_{n}\right)$ are ordered lexicographically.

As mentioned earlier, if two triangular subalgebras of AF algebras (with canonical diagonals) are isometrically isomorphic, then their topologized fundamental relations are isomorphic. We can use this fact to see that no algebra in one of the classes considered above can be isometrically isomorphic to an algebra in another class. Of course, we have already distinguished refinement embeddings from standard embeddings via the respective existence and lack of invariant projections. The alternation embedding may be distinguished from the refinement embedding in precisely the same way while the distinction between alternation and standard is dealt with in [5, Ex. 3.2] with an argument rather more complicated than the one below.

For $x \in M(D)$, define the orbit of $x$ to be $\{y \in M(D) \mid y R(T) x\}$. Orbits and their topological properties are preserved by isomorphism of fundamental relations. The disjointness of the three classes of triangular algebra is apparent from the following orbit structure properties of their fundamental relations. If $T$ is a pure refinement 
algebra, then $R(T)$ has one finite orbit (cardinality $=1$; generator is $x=(1,1,1 \ldots)$ ); one dense orbit (generator is $\left.x=\left(k_{1}, k_{2}, k_{3}, \ldots\right)\right)$; while all remaining orbits are infinite and non-dense. If $T$ is a pure standard algebra, then $R(T)$ has countably many finite orbits (the generators are those $x$ with tails in which all coordinates are 1's), while all remaining orbits are dense. If $T$ is a $\rho \sigma$-alternation algebra, then $R(T)$ has one finite orbit (cardinality $=1$; generator is $x=(\ldots, 1,1,1, \ldots)$ ); uncountably many dense orbits (generators have infinitely many coordinates greater than 1 in negatively indexed locations); and uncountably many infinite, non-dense orbits (generators have at least one coordinate entry greater than 1 while all but finitely many negatively indexed coordinates are equal to 1). All of these facts have completely straightforward verifications.

\section{Classification of $\rho \sigma$-alternation algebras}

We now turn to the determination of the isometric isomorphism classes of the $\rho \sigma$-alternation algebras. Supoose $T$ is the limit of a system

$$
\mathbb{C I} \stackrel{\rho_{r_{1}}}{\longrightarrow} T_{r_{1}} \stackrel{\sigma_{s_{1}}}{\longrightarrow} T_{r_{1} s_{1}} \stackrel{\rho_{r_{2}}}{\longrightarrow} T_{r_{1} s_{1} r_{2}} \rightarrow \cdots
$$

and $S$ is the limit of

$$
\mathbb{C} I \stackrel{\rho_{t_{1}}}{\longrightarrow} T_{t_{1}} \stackrel{\sigma_{u_{2}}}{\longrightarrow} \mathrm{T}_{t_{1} u_{1}} \stackrel{\rho_{t_{2}}}{\longrightarrow} T_{t_{1_{1} u_{1} t_{2}}} \rightarrow \cdots
$$

If $T$ and $S$ are isometrically isomorphic then, by Theorem 1, their enveloping UHF $\mathrm{C}^{*}$-algebras are isomorphic and so we must then have that the supernatural number for the multiplicities associated with $T$-denote by $s n\left(r_{i}, \mathrm{~s}_{i}\right)$-equals $s n\left(t_{i}, u_{i}\right)$, the supernatural number associated with $S$. On the other hand, the supernatural numbers associated with the refinement multiplicities, $\operatorname{sn}\left(r_{i}\right)$ and $\operatorname{sn}\left(t_{i}\right)$, need not be the same since we can modify finitely many embeddings without changing the limit algebra. This turns out to be all the freedom available. Define two supernatural numbers to be finitely equivalent if they differ by finite factors. Let $\sim_{f}$ denote this equivalence relation. With the notation above, we have the following result:

Theorem 2. Let $T$ be a $\rho \sigma$-alternation algebra with refinement multiplicities $r_{i}$ and standard multiplicities $s_{i}$ and let $S$ be another such algebra with refinement multiplicities $t_{i}$ and standard multiplicities $u_{i}$. Then $T$ is isometrically isomorphic to $S$ if, and only if, $\operatorname{sn}\left(r_{i}, s_{i}\right)=\operatorname{sn}\left(t_{i}, u_{i}\right), \operatorname{sn}\left(r_{i}\right) \sim_{f} s n\left(t_{i}\right)$ and $\operatorname{su}\left(s_{i}\right) \sim_{f} s n\left(u_{i}\right)$.

The necessity of the condition on the supernatural numbers will require a way to 


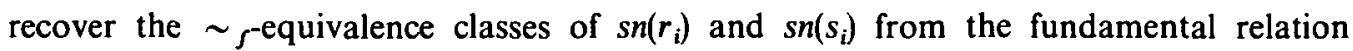
$R(T)$. With $\partial(x)$ denoting the closure of the orbit, $O(x)$, of $x$, the following notion is the key.

Definition. A pair $\left(x, x^{+}\right) \in M(D) \times M(D)$ is a gap pair if $x^{+} \notin \overline{O(x)}$ and $\overline{O\left(x^{+}\right)=}$ $\overline{O(x)} \cup\left\{x^{+}\right\}$.

The first task is to identify which elements $\left(x, x^{+}\right)$form gap pairs. For $x \in M(D)$ and $p>0$, let $M S_{p}(x)=\left(x_{-p}, \ldots, x_{p}\right)$, the symmetric "middle segment" of length $2 p$. For each $x \in M(D)$, a neighbourhood base for $x$ in the Gelfand topology for $M(D)$ is given by the family $N_{p}(x)=\left\{y \mid M S_{p}(y)=M S_{p}(x)\right\}, p=1,2,3, \ldots$ Observe that $y \in \overline{O(x)}$ if, and only if, for any $p>0$ there is an integer $q>p$ such that $M S_{q}(y)<M S_{q}(x)$. (The order is lexicographic.) From this it follows that $\overline{O(x)} \neq M(D)$ (non-dense orbit) if, and only if, there is an integer $p$ such that $x_{q}=1$ for all $q \leqq p$. If $x$ is to be part of a gap pair, it must have non-dense orbit; thus, $x$ must have a left tail consisting of 1's. The next lemma shows that if we add the requirement that $x$ have a right tail consisting of maximal elements, then we will have identifed all the elements $x$ in $M(D)$ which are the left coordinate of a gap pair. The context for this lemma is the fundamental relation $R(T)$ for a $\rho \sigma$-alteration algebra with refinement multiplicities $r_{i}$ and standard multiplicities $s_{i} . M(D)$ is identified with $\pi_{n \neq 0}\left[k_{n}\right]$, where $k_{n}=s_{-n}$ when $n<0$ and $k_{n}=r_{n}$ when $n>0$.

Lemma. If $\left(x, x^{+}\right)$is a gap pair, then $x$ has the following two properties:

(1) There is an integer $n$ such that $x_{m}=1$, for all $m \leqq n$.

(2) There is an integer $p$ such that $x_{q}=k_{q}$ for all $q \geqq p$.

Conversely, suppose that $x$ satisfies (1) and (2) and that $p$ is the smallest integer for which (2) is valid. Define $x^{+}$by

$$
\left(x^{+}\right)_{j}= \begin{cases}x_{j} & \text { if } j<p-1 \\ x_{p-1}+1 & \text { if } j=p-1 \\ 1 & \text { if } j \geqq p\end{cases}
$$

Then $\left(x, x^{+}\right)$is a gap pair.

Proof. We have already seen that condition (1) must hold when $x$ is the left coordinate of a gap pair. Suppose however, that condition (2) is not satisfied. So, for infinitely many $n>0, x_{n} \neq k_{n}$. Suppose that $z \in M(D)$ satisfies $z \notin \overline{O(x)}$ and $x \in \overline{O(z)} \mathrm{We}$ claim that $\overline{O(z)} \backslash \overline{O(x)}$ has infinite cardinality. This is more than enough; even card $[\overline{O(z)} \backslash \overline{O(x)}] \geqq 2$ for all such $z$ shows that $x$ cannot be the left coordinate of a gap pair.

As for the claim, when $z$ does not have a left tail consisting of 1's, we may produce

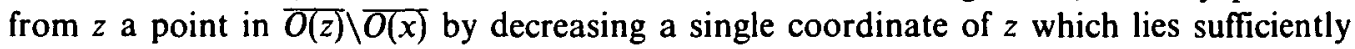
far to the left. Under the hypothesis on $z$ there are infinitely many ways to do this. On the other hand, if $z$ does have a left tail consisting of 1 's, then we can produce from $x$ a

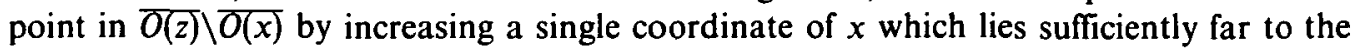


right. Thus, if $\left(x, x^{+}\right)$is a gap pair, $x$ must satisfy both conditions (1) and (2). The converse is straightforward to verify.

Proof of Theorem 2. Assume that $T$ with refinement and standard multiplicities $r_{i}$ and $s_{i}$ and $S$ with refinement multiplicities $t_{i}$ and $u_{i}$ are isometrically isomorphic. As mentioned earlier, it then follows that $s n\left(r_{i}, s_{i}\right)=s n\left(t_{i}, u_{i}\right)$ and $R(T)$ is isomorphic to $R(S)$ as topological binary relations. Furthermore, this order isomorphism is implemented by an order preserving homeomorphism $\psi: M\left(D_{T}\right) \rightarrow M\left(D_{S}\right)$, where $D_{T}$ and $D_{S}$ are the respective canonical masas.

Let $\left(x, x^{+}\right)$be a gap pair in $M\left(D_{T}\right) \times M\left(D_{T}\right)$. Then $\left(\psi(x), \psi\left(x^{+}\right)\right)$is a gap pair in $M\left(D_{S}\right) \times M\left(D_{S}\right)$. The restriction of $\psi$ to $\overline{O(x)}$ induces an order isomorphism from $\overline{O(x)}$ with the relative $R(T)$-fundamental relation to $\overline{O(\psi(x))}$ with the relative $R(S)$ fundamental relation.

Since $x$ is the left coordinate of a gap pair, there are integers $n$ and $p$ such that $x_{m}=1$ for all $m \leqq n$ and $x_{q}=k_{q}$ for all $q \geqq p$. Assume that $n$ is the largest such integer and $p$ the smallest. Also, keep in mind that $k_{j}=s_{j}$ if $j<0$ and $k_{j}=r_{j}$ if $j>0$.

Observe that $y \in \overline{O(x)}$ if, and only if, $y_{m}=1$ for all $m \leqq n$ and $\left(y_{n+1}, \ldots, y_{p-1}\right)<$ $\left(x_{n+1}, \ldots, x_{p-1}\right)$. The right $p$-tail of $y$ is arbitrary. Let $h$ be the cardinality of the set of words preceding, or equal to, $\left(x_{n+1}, \ldots, x_{p-1}\right)$ in $\left[k_{n+1}\right] \times \cdots \times\left[k_{p-1}\right]$. Then $\overline{O(x)} \cong[h] \times$ $\pi_{q \geqq p}\left[k_{q}\right]$ and the relative $R(T)$ order on $\overline{O(x)}$ corresponds to the order in which initial segments are lexicographically ordered and tails are the same, i.e. the pure refinement order with multiplicities $h, k_{p}, k_{p+1}, \ldots$ (If it should happen that $p=n+1$, simply delete $h$ ). Note that $\operatorname{sn}\left(r_{i}\right) \sim_{f} \operatorname{sn}\left(h, k_{p}, k_{p+1}, \ldots\right)$.

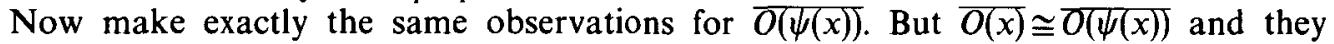
both carry the fundamental relation of a pure refinement algebra, so their associated supernatural numbers are equal. Thus $\operatorname{sn}\left(r_{i}\right)$ and $\operatorname{sn}\left(t_{i}\right)$ are finitely equivalent to the same supernatural number, hence to each other.

It remains to show that $s n\left(s_{i}\right)$ and $s n\left(u_{i}\right)$ are finitely equivalent. We shall write $k_{n}$ and $k_{n}^{\prime}, n \neq 0$, for the multiplicities in the defining systems for $T$ and $S$. It suffices to prove the following fact: there are integers $q$ and $h-1$ such that, for any integer $l<q$ there exists an integer $j<-1$ so that $k_{l}^{\prime} \ldots k_{q-1}^{\prime}$ divides $(h-1) k_{j} \ldots k_{-1}$. (Of course we also need the symmetric fact expressed by interchanging the roles of the sequences $k$ and $k^{\prime}$, but this is obtained by replacing $\psi$ by $\psi^{-1}$ in the argument below.)

Again we shall exploit the facts that $\psi$ preserves closed orbits generated by left coordinates of gap pairs and that such closed orbits with the relative fundamental relation are naturally isomorphic to the fundamental relations of pure refinement algebras. A close orbit of this form is determined by integers $n<m$ and a word $f$ in $\left[k_{n}\right] \times \cdots \times\left[k_{m}\right]$. Denoting the orbit by $[n, m ; f]$ we have $[n, m ; f]=\left\{x \in M(D) \mid x_{i}=1\right.$ for $i<n$ and $\left.\left(x_{n}, \ldots, x_{m}\right) \leqq f\right\}$. Abusing notation somewhat, we shall let $f$ also denote the number of words, $w$, in $\left[k_{n}\right] \times \cdots \times\left[k_{m}\right]$ such that $w \leqq f$ lexicographically.

Let $A=\left[-1,1, k_{1}\right]$. Then $B=\psi(A)=[p, q ; h]$ for some integers $p<q$ and word $h$. Let $l<q$ be given. Without loss of generality, we may assume $l<p$.

Choose an integer $j<-1$ so that any element $x$ in $\psi^{-1}\left[l-1, l ; k_{e}^{\prime}\right]$ has the property that $x_{i}=1$ for all $i \leqq j$. Let $E=\left[j-1, j ; k_{j}\right]$ and let $F=\psi(E)=[v, w ; f]$. Because 
$F \supseteq\left[l-1, l ; k_{e}^{\prime}\right]$, we will have $w \leqq l$. We now have $A \subseteq E, B \subseteq F, \psi(A)=B$ and $\psi(E)=F$. Now view $E$ and $F$ as fundamental relations of appropriate pure refinement algebras. The subsets $A$ and $B$ correspond to projections in the canonical masas and, since $\psi(A)=B$, these projections have the same traces. (The trace in each case is the unique trace in the enveloping UHF $C^{*}$-algebra). Easy calculations yield $\operatorname{tr}(A)=\left(k_{j} \ldots k_{-1}\right)^{-1}$ and $\operatorname{tr}(B)=(h-1)\left(f \cdot k_{w}^{\prime} \ldots k_{q-1}^{\prime}\right)^{-1}$. Here, $f$ and $h$ have the integer interpretation. Thus $f \cdot k_{w}^{\prime} \ldots k_{q-1}^{\prime}=(h-1) k_{j} \ldots k_{-1}$. Since $w<l, k_{l}^{\prime} \ldots k_{q-1}^{\prime}$ divides $(h-1) k_{j} \ldots k_{-1}$, as desired.

For the sufficiency of the conditions on the supernatural numbers, observe first that we may as well assume that $s n\left(r_{i}=s n\left(t_{i}\right)\right.$ and $s n\left(s_{i}\right)=s n\left(u_{i}\right)$. (Limit algebras are unchanged by modifying finitely many embeddings.) The suffiency flows out of the simple observation that $\rho$ and $\sigma$ commute, i.e. $\rho_{r} \sigma_{s}=\sigma_{s} \rho_{r}$, for all $r$, $s$. Then, with $n_{2}=r_{1} s_{1} n_{1}$ and $n_{3}=r_{2} r_{1} s_{2} s_{1} n_{1}=r_{2} s_{2} n_{2}$, the following diagram commutes:

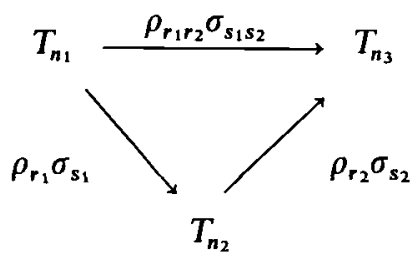

From this, it is a routine matter to construct the usual zig-zag diagram between the systems

$$
\begin{gathered}
T_{n_{1}} \stackrel{\rho_{r_{1}}}{\longrightarrow} T_{n_{2}} \stackrel{\sigma_{s_{1}}}{\longrightarrow} T_{n_{3}} \stackrel{\rho_{r_{2}}}{\longrightarrow} \cdots \\
T_{m_{1}} \stackrel{\rho_{t_{1}}}{\longrightarrow} T_{m_{2}} \stackrel{\sigma_{u_{1}}}{\longrightarrow} T_{m_{3}} \stackrel{\rho_{t_{2}}}{\longrightarrow} \cdots
\end{gathered}
$$

to show that the limit algebras are isometrically isomorphic.

Remarks 1. In [10] it was shown that within a certain family of limits of upper triangular matrix algebras the Banach algebra isomorphism type and the isometric isomorphism type agree (10, Corollary 3.2 and Remark 3.4 ). In view of this, and the fact that this family contains the $\rho \sigma$-alternation limit algebras, Theorem 2 is also valid with isometric isomorphism replaced by Banach algebra isomorphism.

2. During the preparation of this manuscript we learned that Y.-T. Poon [7] has independently obtained Theorem 2 . The method of proof seems to be quite different.

\section{Classification of refinement-twist embeddings}

An important aspect of the $\rho \sigma$-embeddings is that they commute: a composition of refinement and standard embeddings is independent of the order of composition. This 
allowed us to obtain easily the sufficiency condition for isomorphism in the theorem above. Consideration of classes of embeddings without such commutation leads to a much tighter connection between the limit algebra isomorphism type and the ordered sequence of embeddings. We illustrate this rigidity phenomenon with the following theorem. Once more the fundamental relation serves as an effective discriminant.

Write $\tau_{r}: T_{k_{1}} \rightarrow T_{k_{1} r}$ for the unital embeddings of multiplicity $r$ given by $\left(A d U_{k_{1} r}\right) \circ \rho_{r}$ where $U_{s}$ is the permutation unitary in $M_{s}$ which interchanges the last two minimal projections of $D_{s}$. We refer to these as elementary twist embeddings. These embeddings and their limit algebras were introduced by Peters, Poon and Wagner [5]. Notice that the composition $\tau_{r_{1}} \circ \rho_{r_{2}}: T_{k_{1}} \rightarrow T_{k_{1} r_{1} r_{2}}$ coincides with $\tau_{r_{1} r_{2}}: T_{k_{1}} \rightarrow T_{k_{1} r_{1} r_{2}}$ but not with $\rho_{r_{2}} \circ \tau_{r_{1}}$.

Theorem 3. Let $T=\lim T_{n_{k}}$ and $S=\lim T_{m_{k}}$ with respect to elementary twist embeddings. Then $T$ and $S$ are isometrically isomorphic if and only if there is an integer $j$ such that $n_{k+j}=m_{k}$ for all large $k$.

Proof. In order to describe the fundamental relation $R(T)$ and its topology it is helpful to identify the Gelfand space $M\left(D_{T}\right)$ with the Gelfand space of the unital $\mathrm{C}^{*}$-algebra of functions on $[0,1]$ generated by characteristic functions of intervals $[a, b)$ where $a<b$ and $a=i / n_{k}, b=j / n_{i}$ for some $i, j, k, l$. In particular there are natural maps $\pi: M\left(D_{T}\right) \rightarrow[0,1], \pi^{(2)}: R(T) \rightarrow[0,1] \times[0,1]$. The map $\pi$ is surjective. Notice that $T$ and the pure refinement algebra $A=\lim \left(T_{n_{k}}, \rho_{k}\right)$, where $\rho_{k}$ has multiplicity $r_{k}=n_{k+1} / n_{k}$, $k=1,2, \ldots$, have a common subalgebra $T_{0}=\lim T_{n_{k}}^{0}$. Here, $T_{n_{k}}^{0} \subset T_{n_{k}}$ is the unital subalgebra $(1-p) \mathbb{C}+T_{n_{k}} p$, where $p$ is the largest proper projection of $L$ at $T_{n_{k}}$ (the invariant projection lattice). It follows that $\pi^{(2)}(R(T))$ contains $\pi^{(2)}\left(R\left(T_{0}\right)\right)$, and this set is the union of the appropriate rational superdiagonals of $[0,1) \times[0,1)$ together with the diagonal $\Delta=\{(t, t): t \in[0,1]\}$.

Every compact, open set $E$ in $R(T)$ corresponds to a finite union of graphs $E(v)$ associated with the canonical matrix units $v$ (see [9]). Here $E(v)$ is the set of pairs $(y, x)$ with $y=\alpha(x), \alpha$ is the partial homomorphism induced by $v$ (as described in the introduction), and $x$ is a point of the domain of $\alpha$. If $e_{1 n_{1}}$ in $T_{n_{1}}$ has image $v$ in $T$ then it is elementary to verify that $\pi^{(2)}(E(v))$ is the set suggested by the following diagram:

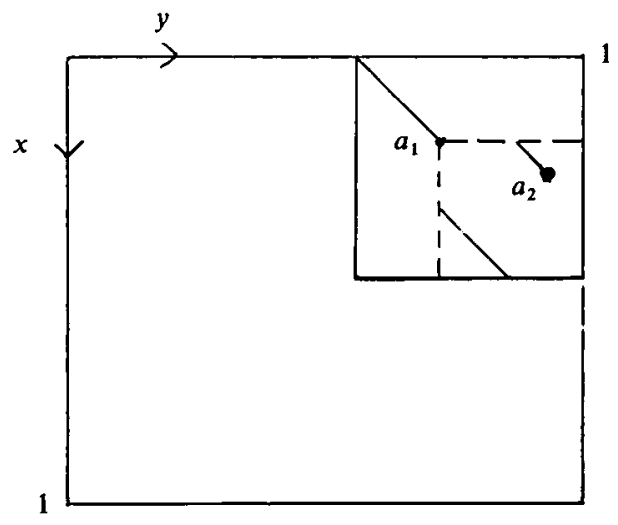


In the diagram

$$
a_{1}=\left(\frac{r_{1}-2}{n_{1} r_{1}}, \frac{n_{1}-1}{n_{1}}+\frac{r_{1}-2}{n_{1} r_{1}}\right)
$$

and $a_{k}$ is defined inductively by

$$
a_{k}=a_{k-1}+\left(\frac{r_{k}-2}{n_{k+1}}, \frac{1}{n_{k}}+\frac{r_{k}-2}{n_{k+1}}\right) .
$$

Thus, $\pi^{(2)}(E(v))$ is the union of the closed diagonal segments of the diagrams

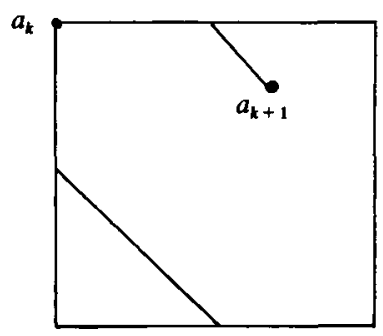

for $k=1,2, \ldots$, together with the initial segment from $\left(0,1-\frac{1}{n_{1}}\right)$ to $a_{1}$, and the limit point, $(p, 1)$, say.

Similarly, any matrix unit $w$ in $T_{n_{k}} \backslash T_{n_{k}}^{0}$ has a graph $\pi^{(2)}(E(w))$ which is eventually congruent to $\pi^{(2)}(E(v))$. More precisely, there exists $s$ in $(0,1)$ such that $([0,1] \times[1-s, 1]) \cap \pi^{(2)}(E(v))$ is a vertical translate of $([0,1] \times[1-s, 1]) \cap \pi^{(2)}(E(w))$.

Suppose now that $\phi: T \rightarrow S$ is an isometric isomorphism. Then $\phi$ extends to a star isomorphism $\bar{\phi}: C^{*}(T) \rightarrow \mathrm{C}^{*}(S)$ (by [6], or [2]) and so $\phi$ preserves the unique normalised traces on $T$ and $S$. Also $\phi\left(\widetilde{T}_{0}\right)=S_{0}$ (by the proof of Theorem 1). If $\theta: R\left(T_{0}\right) \rightarrow R\left(S_{0}\right)$ is the induced topological isomorphism it follows that we have a commuting diagram:

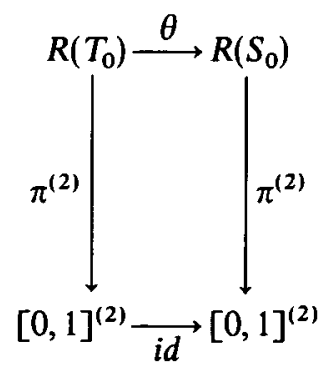

Let $v^{\prime}$ be the image of $e_{1 m_{1}}$ in $S$. Since $v^{\prime}$ is a normalising partial isometry in $S \backslash S_{0}$, 
$\phi^{-1}\left(v^{\prime}\right)$ must be a normalising partial isometry in $T \backslash T_{0}$, and so $\pi^{(2)}\left(E\left(\phi^{-1}\left(v^{\prime}\right)\right)\right)$ is eventually congruent to $\pi^{(2)}(E(v))$. But, because of the commuting diagram this means that the sequence $\left(a_{k}\right)$ for $v$ is eventually congruent to the sequence $\left(a_{k}^{\prime}\right)$, say, for $v^{\prime}$, by a vertical translation. The desired conclusion now follows.

Remark. It is not difficult to indentify the fundamental relation for the twisted refinement limit algebra with multiplicites $k_{1}, k_{2}, \ldots$ as it appears in the $\pi_{n=1}^{\infty}\left[k_{n}\right]$ representation. For most matrix units $e_{i j}$ in $T_{k_{1} \ldots k_{n}}, \tau\left(e_{i j}\right)=\rho\left(e_{i j}\right)$. This equality fails to hold only when $j=\left(k_{1}, \ldots, k_{n}\right)$. In this case, $\rho\left(e_{i j}\right)$ is a sum of $k_{n+1}$ matrix units in $T_{k_{1} \ldots k_{n+1}}$ of which two of the terms in the sum must be modified to produce $t\left(e_{i j}\right)$. The summands of $\rho\left(e_{i j}\right)$ have multi-indices $(i, h)$ and $(j, h)$, for $h=1, \ldots, k_{n+1}$ (where we write $i$ for $\left(i_{1}, \ldots, i_{n}\right)$, etc.). The summands to be modified are the last two, corresponding to the action of the twist on the last two columns. The two replacement partial isometries have indices $\left(i, k_{n+1}\right),\left(j, k_{n+1}-1\right)$ and $\left(i, k_{n+1}-1\right),\left(j, k_{n+1}\right)$.

Tracking $e_{i j}$ forward under subsequent embeddings allows the identification of the corresponding partial homeomorphism on $M(D)$. The domain is the set of all sequences in $M(D)$ with initial segment $j$. If at least one $j_{p} \neq k_{p}$, then the partial homeomorphism acts just as it does in the pure refinement setting. When $j_{1}=k_{1}, \ldots, j_{n}=k_{n}$, the action on a point $\left(k_{1}, \ldots, k_{n}, x_{n+1}, \ldots\right)$ can be described intuitively as follows: the first $n$ coordinates are converted to $i_{1}, \ldots, i_{n}$. Now look at the tail. If it begins with a coordinate $\leqq k_{n+1}-2$, the whole tail is unmodified. If it begins with $k_{n+1}-1$, then this term is changed to $k_{n+1}$ and the rest of the tail is unmodified. If the tail begins with a string of maximal terms, each of these is decreased by 1 ; the first non-maximal term, $x_{m}$, is unchanged unless it is equal to $k_{m}-1$, in which case it is increased by 1 . Subsequent terms are unchanged. If the tail consists entirely of maximal terms, each is decreased by 1 .

Although the partial homeornorphisms induced by $e_{i j}$ are different from those induced in the refinement case (when $j_{1}=k_{1}, \ldots, j_{n}=k_{n}$ ), all but one point of the graph of $e_{i j}$ in the twisted embedding lies in the graph of some matrix unit in the refinement embedding.

With $T$ the twisted refinement limit algebra, we then have the following description of $R(T)$ : If some $x_{n} \neq k_{n}$, then $y R(T) x$ if and only if $y$ and $x$ have identical tails and the initial segment of $y$ precedes the initial segment of $x$ lexicographically. If $x_{n}=k_{n}$ for all $n$, $y R(T) x$ if and only if $y_{m}=k_{m}-1$, for all $m$ sufficiently large. These latter points are the limit points on the right hand edge in the $[0,1] \times[0,1]$ representation.

\section{Classification of some homogeneous limit algebras}

If $m=n r$, define $\phi_{r}: T_{m} \rightarrow T_{n r}$ by $\phi\left(a_{i j}\right)=\left(a_{i j} U_{r}^{j-i}\right)$ where $U_{r}$ in $M_{r}$ is the cyclic backward shift. For convenience we call such embeddings homogeneous embeddings; the associated limit algebras $T$ have the homogeneous property that subalgebras $p T p, q T q$ determined by interval projections $p, q$ of equal trace, are isometrically isomorphic. We shall obtain the following rigidity theorem by examining the fractal-like subsets of the square arising as support sets for normalising partial isometries, as in the last section. Of course one 
could consider, more generally, homogeneous limit algebras relative to a sequence of arbitrary permutation unitaries, but we focus here on the purely cyclic case.

Theorem 4. Let $T=\lim T_{n_{k}}$ and $S=\lim T_{m_{k}}$ with respect to homogeneous embeddings (associated with backward cyclic shifts). Then $T$ and $S$ are isometrically isomorphic if and only if there is an integer $j$ such that $n_{k+j}=m_{k}$ for all large $k$.

Proof. As before, the trace functional gives rise to natural projections $\pi: M\left(D_{T}\right) \rightarrow$ $[0,1], \pi^{(2)}: R(T) \rightarrow[0,1]^{(2)}$, with similar maps for $M\left(D_{S}\right)$ and $R(S)$. Assume that $T$ and $S$ are isometrically isomorphic by an isomorphism $\alpha: T \rightarrow S$. Once again, since trace is preserved, we obtain a commuting diagram:

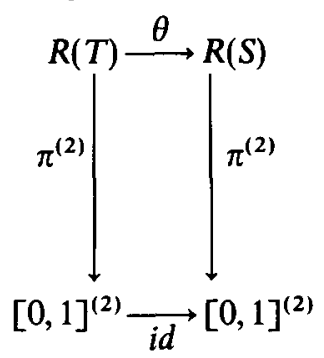

Let $e_{i, i+1}$ be a superdiagonal matrix unit in $T_{n_{k}}$ with image $v$ in $T$, and graph $E(v) \subset R(T)$. Then the set $E=\pi^{(2)}(E(v))$ is an intersection $E_{1} \cap E_{2} \cap \cdots$ where $E_{1}$ is the square of side $1 / n_{k}$ in the $i, i+1$ location of the $n_{i}^{2}$ refinement of $[0,1]^{(2)}$, and $E_{k+1} \subset E_{k}$ is the finite union of $r_{k}=n_{k+1} / n_{k}$ subsquares of sidelength $1 / n_{k+1}$ located in one of the $r_{k}$ possible cyclic patterns. Note in particular that each $E_{k}$ contains a superdiagonal subsquare, $E_{k}^{s}$ say, and that $E_{k+1} \cap E_{k}^{s}$ is always in the backward cyclic shift pattern. The following diagram illustrates two generations of subsquares of a given square of $E_{k}$ :

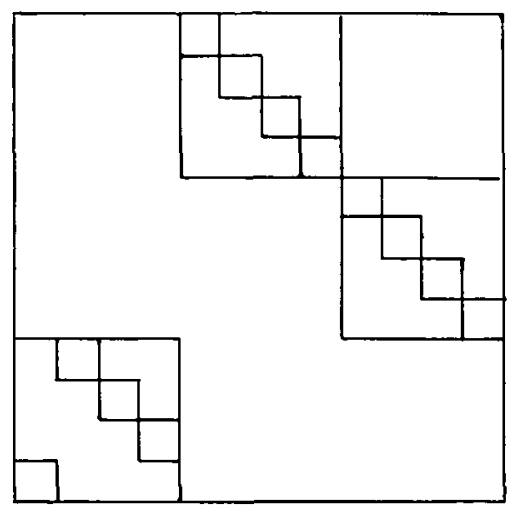

Recall that every normalising partial isometry is a sum of matrix units, modulo unitary multipliers from the diagonal ([8, Lemma 6.3]). It follows from this, and the commuting diagram, that $\pi^{(2)}(E(v))=\pi^{(2)}(E(\alpha(v)))$ is a disjoint union of sets $E\left(w_{1}\right), \ldots, E\left(w_{k}\right)$ associated with matrix units $w_{1}, \ldots, w_{k}$ in $T_{j}$ for some $j$.

We claim that such an equality $E=E\left(w_{1}\right) \cup \cdots \cup E\left(w_{k}\right)$ is only possible if the scaling 
dimensions $\left(n_{k}\right)$ and $\left(m_{k}\right)$ are asymptotically equal. Here is one way to see this geometric fact.

Note first that if $n_{k+1} / n_{k}=2$ for all $k \geqq k_{0}$, for some $k_{0}$, then $E$ is a union of closed line segments, and in the alternative case $E$ is a set containing no line segments. However, in the former case if $T$ and $S$ are isometrically isomorphic then they have the same supernatural number invariant, namely $l 2^{\infty}$ for some natural number $l$, and the conclusion of the theorem follows trivially in this case. Accordingly we may assume the latter "fractal" case for both $\left(n_{k}\right)$ and $\left(m_{k}\right)$.

If $F$ is a subsquare of the unit square, with vertices with rational coordinates, then say that $F_{1}, \ldots, F_{r}$ is a permutation covering of $F$ if $F_{1}, \ldots, F_{r}$ are congruent closed squares such that for some permutation $\pi$ of $\{1, \ldots, r\}, F_{i}$ is the square of the $r^{2}$ refinement of $E$ located in the $i, \pi(i)$ position. Call the reciprocal of the sidelength of a set $F_{i}$ the size of the permutation cover. All squares are assumed to have sides parallel to those of the unit square. For a given square $F$ the set of sizes of permutation coverings is an intrinsic property of $F$. We leave it as an elementary exercise to show that for the set $E$ the set of permutation covering sizes is precisely $\left\{n_{k}, n_{k+1}, \ldots\right\}$. Since $E$ comes from a superdiagonal matrix unit it follows that one of $w_{1}, \ldots, w_{k}$ is a superdiagonal matrix unit in $T_{m}, S$. Its permutation covering sizes are $m_{j}, m_{j+1}, \ldots$, and so the desired conclusions follows.

Remark. In each of the proofs above we have made key use of the fact that the topologised fundamental relation $R(A)$ is an invariant for isometric isomorphism and that this topology detects the essential algebraic structure of the algebra. We remark that if $R(A)$ is viewed simply as a binary relation on a topological space, then it does not determine isomorphism type. More precisely, there exist non isomorphic triangular subalgebras $A, A^{\prime}$ of $\mathrm{AF} \mathrm{C}^{*}$-algebras, with canonical subalgebras $C, C^{\prime}$, such that there exists a homeomorphism $\theta: M(C) \rightarrow M\left(C^{\prime}\right)$ with $\theta^{(2)}(R(A))=R\left(A^{\prime}\right)$. To see this consider the algebra $A_{0}$ of compact operators on $l^{2}(\mathbb{Z})$ which have upper triangular representing matrices with respect to the standard bases $\left\{e_{n}: n \in \mathbb{Z}\right\}$. Let $P_{+}, P_{-}$be the orthogonal projections onto $l^{2}\left(\mathbb{Z}_{+}\right), l^{2}\left(\mathbb{Z}_{-}\right)$respectively, where $\mathbb{Z}_{+}$and $\mathbb{Z}_{-}$are the nonnegative and negative integers, and let $U$ be a partial isometry on $l^{2}(\mathbb{Z})$ with initial space $l^{2}\left(\mathbb{Z}_{+}\right)$and final space $l^{2}\left(\mathbb{Z}_{-}\right)$such that $U_{e_{n}}=e_{\pi(n)}$ for some bijection $\pi: \mathbb{Z}_{+} \rightarrow \mathbb{Z}_{-}$. Then the algebra $A=\tilde{A}_{0}+\mathbb{C} U$, with $\tilde{A}_{0}=\mathbb{C} P_{+}+\mathbb{C} P_{-}+A_{0}$, is a canonical triangular subalgebra of an AF $\mathrm{C}^{*}$-algebra. The fundamental relation, as a binary relation on the Gelfand space $M\left(A \cap A^{*}\right)=\{-\infty\} \cup \mathbb{Z} \cup\{+\infty\}$, is independent of the choice of $\pi$, and is simply the total order on $\mathbb{Z}$ together with the points $(-\infty,-\infty),(-\infty,+\infty),(+\infty,+\infty)$. On the otherhand it is straightforward to show that two such algebras, associated with $\pi, \pi^{\prime}$ say, are isometrically isomorphic if and only if there is an integer $j$ such that $\pi(n)=\pi(n+j)$ for all large $n$ in $\mathbb{Z}_{+}$.

\section{REFERENCES}

1. R. L. BaKer, Triangular UHF algebras, J. Funct. Anal. 91 (1990), 182-212.

2. K. R. Davidson and S. C. Power, Isometric automorphisms and homology for non-selfadjoint operator algebras, Quart. J. Math. 42 (1991), 271-292. 
3. J. Gumm, On a certain class of operator algebras, Trans. Amer. Math. Soc. 95 (1960), 318-340.

4. P. S. Munly and B. Solel, On triangular subalgebras of groupoid $\mathrm{C}^{*}$-algebras, preprint 1989.

5. J. R. Peters, Y.-T. Poon and B. H. WAGner, Triangular AF algebras, J. Operator Theory 23 (1990), 81-114.

6. J. R. Peters and B. H. Wagner, Triangular AF algebras and nest subalgebras of UHF algebras, J. Operator Theory, to appear.

7. Y.-T. Poon, A complete isomorphism invariant for a class of triangular UHF algebras, $J$. Operator Theory, to appear.

8. S. C. POWER, Classifications of tensor products of triangular operator algebras, Proc. London Math. Soc. 61 (1990), 571-614.

9. S. C. POWER, The classification of triangular subalgebras of $\mathrm{AF} \mathrm{C}^{*}$-algebras, Bull. London Math. Soc. 22 (1990), 269-272.

10. S. C. Power, Algebraic orders on $K_{0}$ and approximately finite operator algebras, $J$. Operator Theory, to appear.

11. S. Stratila and D. Voiculescu, Representations of AF-algebras and the group $U(\infty)$ (Lecture Notes in Mathematics, 486, Springer-Verlag, New York, 1975).

12. M. A. Thelwall, Maximal triangular subalgebras of AF algebras, $J$. Operator Theory, to appear.

13. M. A. Thelwall, Dilation theory for subalgebras of AF algebras, J. Operator Theory, to appear.

14. B. A. Ventura, Strongly maximal triangular AF algebras, Internat. J. Math. 2 (1991), $567-598$.

Department of Mathematics

UNIVERSITY OF Alabama

TUSCALOOSA, AL 35487

USA
Department of Mathematics

UNIVERSITY OF LANCASTER

LANCASTER LAI 4YL

ENGLAND 